\title{
PHYSIOLOGICAL RESPONSES DURING COMPETITIVE SPORTS AEROBICS EXERCISE
}

\author{
Roma Aleksandravičienė, Arvydas Stasiulis \\ Lithuanian Academy of Physical Education, Kaunas, Lithuania
}

\begin{abstract}
Roma Aleksandravičienė. PhD student of biology at the Lithuanian Academy of Physical Education, assistant of Gymnastics and Dance Department. The field of scientific research — aerobic performance's acute and chronic adaptation to the influence of training and laboratory loads.
\end{abstract}

\begin{abstract}
The aim of this study was to characterize heart rate (HR), oxygen uptake and pulmonary ventilation during competitive aerobic gymnastics routine in a group of elite women athletes. The subjects were Lithuanian aerobic women gymnasts, members of national team $(21.6,4.4)$ years old). All subjects performed a maximal incremental treadmill test in the laboratory and competitive aerobic gymnastics exercises in group category. Heart rate was continuously recorded using the heart rate measurement equipment Polar ACCUREX-Plus. During the incremental treadmill test HR deflection point and other parameters of aerobic capacity were determined from the relationship of HR to running speed. During the aerobic gymnastics routine pulmonary gas exchange parameters and heart rate were continuously measured using the telemetric equipment Cortex $3 B$. The changes of HR, minute ventilation and oxygen uptake were analyzed by adopting monoexponential function.

The results showed that HR values during the competitive aerobic gymnastics routine were higher than HR break point which is near the lactate accumulation threshold (reaching 95.2 (4.2)\% of maximal HR). Oxygen uptake during competitive routine reached 81.3 (5.8)\% of maximal oxygen uptake. Rather high blood lactate accumulation $(7.50 \mathrm{mmol} / \mathrm{l}$ ) at the third minute after exercise show the high intensity of exercise. These results allows us to consider that aerobic gymnastics is a sport with high cardiorespiratory and metabolic demands, in which aerobic and anaerobic sources are intensely activated.
\end{abstract}

Keywords: aerobic gymnastics, aerobic capacity, pulmonary gas exchange, lactate, heart rate deflection point.

\section{INTRODUCTION}

1 erobic gymnastics became a member of the gymnastics family, and thus of the International Gymnastics Federation in 1994. This new kind of sport increased in popularity after $1^{\text {st }}$ Sport Aerobics World Championship, which was held in Paris, in 1995. The competition in aerobic gymnastics includes the routine lasting on average $1 \mathrm{~min} 45 \mathrm{~s}$. It is a sport with the unique structure of the routine, with a different degree of difficulty elements, complex movements patterns and steps in linking to music. All the time a participant must move in time following the beat of music and musical phrases, it means - without any phase of rest. All movements, including difficulty elements, must be performed with correct posture and with body alignment (neutral alignment without hyperextension of joints).

The physiological responses of aerobic gymnastics exercise have not yet been widely studied. Only one short study has been published describing physiological characteristics of aerobic gymnasts and physiological responses during competitive routine (Rodriguez et al., 1998). Currently measurements of pulmonary gas exchange and heart rate can be continuously 
obtained using telemetric systems (Schulz et al., 1997; Maiolo et al., 2003) and metabolic profile based on the measurement of the blood lactate concentration and oxygen consumption pre, during and post different kinds of acyclic activities can be established (Guidetti et al., 2000; Beneke et al., 2004). As a member of gymnastics family aerobic gymnastics in some aspects can be comparable with other kinds of gymnastics sport. Several studies have been published describing the characteristics of rhythmic gymnasts (Case et al., 1980; Gionet et al., 1986; Alexander, 1991) and energy requirement of ball routine in rhythmic gymnastics (Guidetti et al., 2000).

The aim of this study was to characterize heart rate (HR), oxygen uptake and pulmonary ventilation during competitive aerobic gymnastics routine in a group of elite women athletes.

\section{METHODS}

Subjects. 9 Lithuanian aerobic women gymnasts participated in this study. Age and physical characteristics of gymnasts are presented in Table 1. All subjects were members of national team and they were tested during their competitive period before the World Championship.

All athletes' participation in the study was requested through their coaches. The informed consent was obtained from all participants. The study was approved by Regional Ethics Committee for biomedical research.

Experimental procedure. All subjects underwent two testing sessions. Both testing sessions were performed one week apart. All athletes were asked not to train vigorously on the eve before both testings. During the first session (treadmill test) the parameters of $\mathrm{VO}_{2} \max , \mathrm{HR}$ deflection point were estimated. In the second session (aerobic gymnastics routine) pulmonary gas exchanges parameters, heart rate and blood lactate concentration were measured.

First test session. All subjects performed a maximal incremental treadmill test in the

Table 1. Anthropometric characteristics and age of aerobic gymnasts (mean (SD))

\begin{tabular}{|l|c|}
\hline Age, years & $21.6(4.4)$ \\
\hline Height, $\mathrm{cm}$ & $154(5.0)$ \\
\hline Body mass, kg & $55.1(6.2)$ \\
\hline
\end{tabular}

laboratory. Before the test each athlete performed a 5 min. warm up, which consisted of walking, easy running and stretching exercises. The treadmill test consisted of each minute increasing running. Starting workload was $6.2 \mathrm{~km} / \mathrm{h}$, each minute running speed increased by $0.7 \mathrm{~km} / \mathrm{h}$. All gymnasts were encouraged to continue as long as they were able to maintain speed of running. Heart rate was continuously recorded using the heart rate measurement equipment Polar ACCUREX-Plus. The HR deflection point and other parameters of aerobic capacity were determined from the relationship of HR to running speed.

Second testing session. Over one week, the athletes performed competitive aerobic gymnastics exercises in group category. During the routine pulmonary gas exchange parameters and heart rate were continuously measured using the telemetric equipment Cortex $3 B$. The changes of HR, minute ventilation and oxygen uptake were analyzed by adopting monoexponential function. The heart rate was recorded continuously every $5 \mathrm{~s}$ using the Polar heart rate monitor.

The length of routine was $1 \mathrm{~min} 46 \mathrm{~s}$. The aerobics gymnastics exercises must be performed under artistic, execution and difficulty criterions. The main artistic criterions are: choreography, sports specific content, musicality and expression, execution criterions - technical execution of all movements including difficulty elements. The main difficulty criterion is the performance of one minimum element (maximum 12 elements are allowed) from each group of element pool (A dynamic strength, $\mathrm{B}$ - static strength, $\mathrm{C}$ - jumps, $\mathrm{D}$ - flexibility). Each difficulty element is evaluated under the level of difficulty. The subjects performed the routine that consisted of 12 elements with difficulty value of 4.0 points (Federation Internationale de Gymnastique, 2002).

Before, during and $10 \mathrm{~min}$ after the routine pulmonary gas exchange parameters and HR were continuously measured using the telemetric equipment Cortex 3B. Capillary blood samples were obtained before the routine and at the end of, the third and $18^{\text {th }}$ mins of recovery and analysed for blood lactate concentration (Kulis et al., 1988).

Data analysis. During a maximal incremental treadmill test $\mathrm{HR}$ deflection point and $\mathrm{VO}_{2}$ parameters of aerobic capacity were determined from the relationship of HR to running speed. 


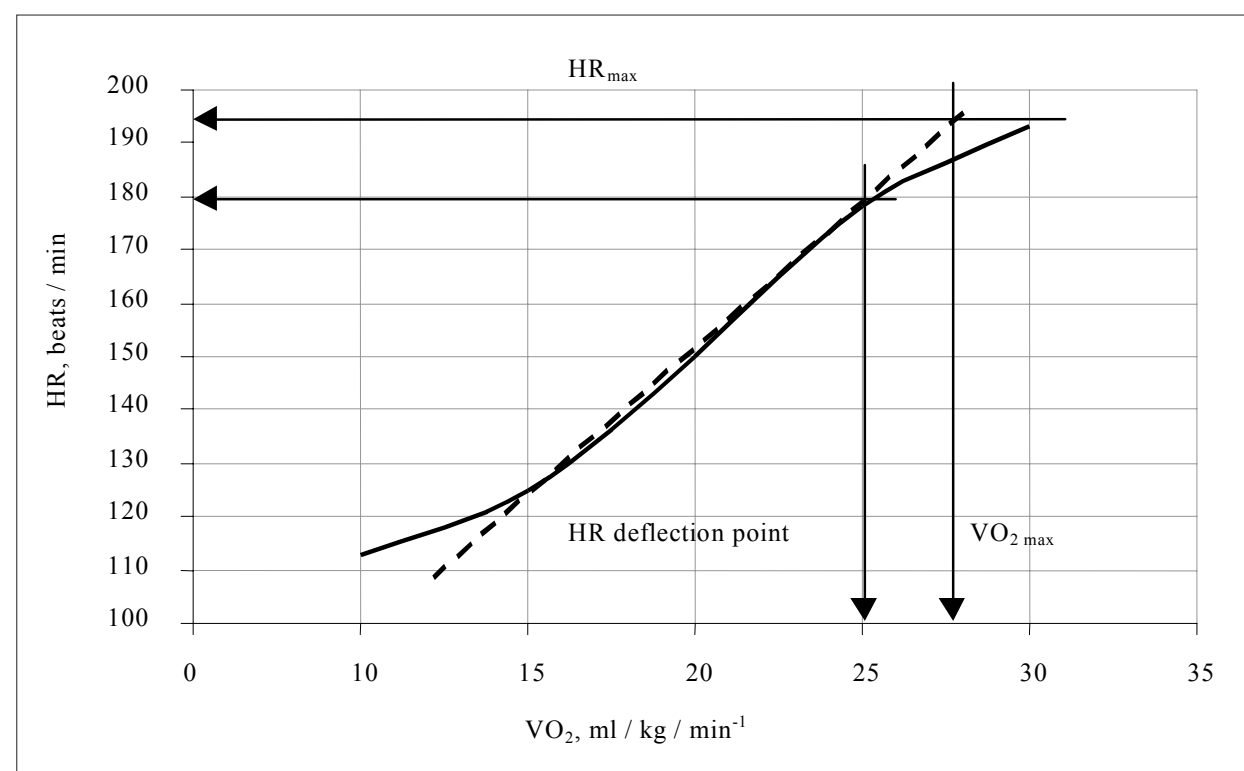

Fig. 1. Relationship of heart rate to running speed in one subject

During the competitive aerobics gymnastics routine the changes of HR, minute ventilation and oxygen uptake were analyzed by adopting monoexponential function.

Statistical analysis. Means' values, standard deviations and correlation were calculated.

\section{RESULTS}

Maximal responses during the incremental test on the treadmill are presented in Table 2. Sports aerobics athletes have a moderate maximal aerobic power. The competitive aerobic gymnastics routine are characterized by very intense cardiorespiratory demands, attaining

\begin{tabular}{|l|c|}
\hline $\mathrm{HR}_{\max }$, beats $/ \mathrm{min}^{-1}$ & $193.3(7.7)$ \\
\hline $\mathrm{HR}$ deflection point, $\mathrm{km} / \mathrm{h}^{-1}$ & $10.9(0.8)$ \\
\hline $\mathrm{HR}$ at deflection point, beats $/ \mathrm{min}^{-1}$ & $171.1(5.9)$ \\
\hline Maximal aerobic speed, $\mathrm{km} / \mathrm{h}^{-1}$ & $13.31(0.87)$ \\
\hline $\mathrm{VO}_{2 \max }, 1 / \mathrm{min}^{-1}$ & $2.78(0.38)$ \\
\hline $\mathrm{VO}_{2 \max }, \mathrm{ml} / \mathrm{kg} / \mathrm{min}^{-1}$ & $50.38(1.51)$ \\
\hline
\end{tabular}

Table 2. Maximal responses in aerobic gymnasts during an incremental treadmill test (mean (SD))

\begin{tabular}{|l|c|}
\hline HR asymptote, beats $/ \mathrm{min}^{-1}$ & $182.1(7.5)$ \\
\hline HR time constant, $\mathrm{s}$ & $16.5(5.6)$ \\
\hline Minute ventilation asymptote, $1 / \mathrm{min}^{-1}$ & $82.4(17.8)$ \\
\hline Minute ventilation time constant, $\mathrm{s}$ & $49.4(17.7)$ \\
\hline $\mathrm{VO}_{2}$ asymptote, $1 / \mathrm{min}^{-1}$ & $2.34(0.22)$ \\
\hline $\mathrm{VO}_{2}$ time constant, $\mathrm{s}$ & $30.9(15.7)$ \\
\hline$[$ Lactate $]$ at $3^{\text {rd }}$ min after exercise, $\mathrm{mmol} / 1$ & $7.50(2.09)$ \\
\hline$[$ Lactate $]$ at $18^{\text {th }}$ min after exercise, $\mathrm{mmol} / 1$ & $4.99(2.30)$ \\
\hline
\end{tabular}

Table 3. Cardiorespiratory values during a competitive aerobic gymnastics routine (mean (SD)) 
Fig. 2. Heart rate, minute ventilation and oxygen consumption during competitive gymnastics aerobic routine in one subject

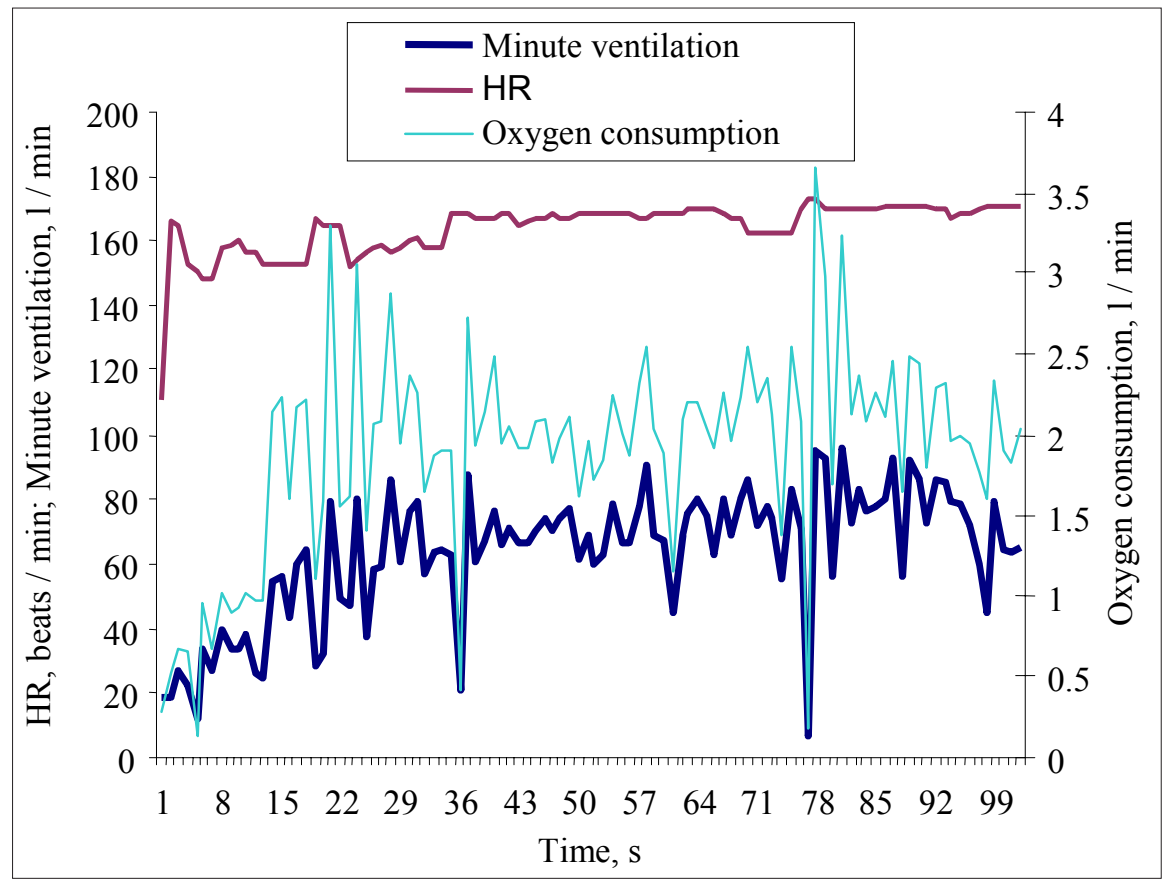

average maximal values $81.3(5.8) \%$ of maximal oxygen uptake and reaching $95.2(4.2) \%$ of maximal HR and overstepping the HR break point which is near the lactate accumulation threshold (Table 2, Fig. 1). A high correlation was observed between $\mathrm{VO}_{2}$ max determined in the laboratory and $\mathrm{VO}_{2}$ measured during the routine $(\mathrm{r}=0.872$; $\mathrm{p}<0.05)$.

\section{DISCUSSION}

The aerobic capacity and physiological responses during competitive routine in well trained aerobic gymnasts were analyzed. Also, the correlation between all parameters mentioned above was assessed.

Maximal responses during treadmill test in Spanish athletes have been published (Rodriguez et al., 1998). $\mathrm{HR}_{\max }$ in their study was similar. Absolute $\mathrm{VO}_{2}$ peak data were higher in $\mathrm{F}$. A. Rodriguez's et al. (1998) study in comparison with our calculated data. Spanish gymnasts were of elite international level while ours only of moderate one. This also might be due to different body composition. Our gymnasts were taller and heavier than Spanish ones. In addition, Spanish athletes seem to have higher anaerobic capacity since they achieved higher blood lactate concentration after competitive routine.

Anaerobic thresholds had not been previously evaluated in aerobic gymnasts, but they were evaluated in rhythmic gymnasts (Baldari, Guidetti, 2001). In the present study we did measure only HR deflection point which is related to blood lactate accumulation threshold.

Only maximal values of physiological variables during competitive routine were presented in the Spanish study (Rodriguez et al., 1998), so all variables in there study were higher in comparison with our asymptotic values. The single and trios were tested in the Spanish study and it is known that this kind of aerobic gymnastics is more intensive. This might be the reason of higher lactate concentration in F. A. Rodriguez's et al. (1998) study.

In this study HR during the quasi-steady state of competitive exercise was higher than at HR deflection point. This may be associated with rather high blood lactate levels ( 7.50 at the $3 \mathrm{rd}$ min after exercise). The marked activation of the cardiorespiratory system may be associated with the involvement of whole body muscles during aerobics exercise. Aerobics can be considered as continuous exercise involving different types of contraction by different muscle groups The specific training of aerobic gymnastics appears to yield a fitness structure with relatively more developed aerobic and anaerobic alactic capacities and less developed anaerobic lactatic capacity.

\section{CONCLUSIONS}

Competitive aerobic gymnastics exercise can be considered as a sport with high cardiorespiratory and metabolic demands, in which aerobic and anaerobic sources are intensely activated. 


\section{REFERENCES}

Alexander, M. J. (1991). Physiological characteristics of elite and subelite rhythmic gymnastics. Journal of Human Movement Studies, 20, 99-127.

Baldari, C., Guidetti, L. (2001). $\mathrm{VO}_{2} \max$, ventilatory and anaerobic thresholds in rhythmic gymnasts and young female dancers. Journal of Sports Medicine and Physical Fitness, 41, 177-82.

Beneke, R., Beyer T., Jachner, C., Erasmus, J., Hutler, M. (2004). Energetics of karate kumite. European Journal of Applied Physiology, 92, 318-523.

Case, S., Fleck, S., Koehler, P. (1980). Physiological and performance characteristics of the 1979 US MRG team. International Gymnast, 26, 4.

Gionet, N., Babineau, C., Brjant, D. (1986). Physiological evaluation of elite Canadian rhythmic sportive gymnasts (RSG) (Abstract). Canadian Journal of Applied Sport Sciences, 11, 15.

Guidetti, L., Baldari, C., Capranica, C., Persichini, C., Figura, F. (2000). Energy cost and energy sources of ball routine in rhythmic gymnasts. International Journal of Sports Medicine, 21, 205-209.

Federation Internationale de Gymnastique. (2002). Sports Aerobics Code of Points 2001-2004. Moutier.

Kulis, Y., Laurinavichyus, V., Firantas, A., Kurtinaitienė, B. (1988). Determination of lactic acid with an Exan-G analyser. Journal of Analytical Chemistry of the USSR, 43 (7), 1521-1523.

Maiolo, C., Melchiorri, G., Iacopino, L., Masala, S., De Lorenzo, A. (2003). Physical activity energy expenditure measured using a portable telemetric device in comparison with a mass spectrometer. Journal of Sports Medicine, 37 (5), 445-447.

Rodriguez, F. A., Iglesias, X., Marina, M., Fado, C. (1998). Physiological demands of elite competitive aerobics. Journal of Sports Science, 16, 510-511.

Schulz, H., Helle, S., Heck, H. (1997). The validity of the telemetric system Cortex X1 in the ventilatory and gas exchange measurement during exercise. International Journal of Sports Medicine, 18, 454-457.
Received on May 13, 2005

Accepted on June 30, 2005
Roma Aleksandravičienè

Lithuanian Academy of Physical Education

Sporto str. 6, LT-44221 Kaunas

Lithuania

Tel +37037302639

E-mail.gsk@1kka.lt 